This item was submitted to Loughborough's Research Repository by the author.

Items in Figshare are protected by copyright, with all rights reserved, unless otherwise indicated.

\title{
Robust nonlinear generalised predictive control for a class of uncertain nonlinear systems via an integral sliding mode approach
}

PLEASE CITE THE PUBLISHED VERSION

http://dx.doi.org/10.1080/00207179.2016.1145356

\section{PUBLISHER}

(c) Taylor \& Francis

\section{VERSION}

AM (Accepted Manuscript)

\section{PUBLISHER STATEMENT}

This work is made available according to the conditions of the Creative Commons Attribution-NonCommercialNoDerivatives 4.0 International (CC BY-NC-ND 4.0) licence. Full details of this licence are available at: https://creativecommons.org/licenses/by-nc-nd/4.0/

\section{LICENCE}

CC BY-NC-ND 4.0

\section{REPOSITORY RECORD}

Errouissi, Rachid, Jun Yang, Wen-Hua Chen, and Ahmed Al-Durra. 2016. "Robust Nonlinear Generalised Predictive Control for a Class of Uncertain Nonlinear Systems via an Integral Sliding Mode Approach". figshare. https://hdl.handle.net/2134/20890. 


\title{
Robust Nonlinear generalized predictive control for a class of uncertain nonlinear systems via an integral sliding mode approach
}

\author{
Rachid Errouissi ${ }^{a *}$, Jun Yang $^{b}$, Wen-Hua Chen ${ }^{c}$ and Ahmed Al-Durra ${ }^{a}$ \\ ${ }^{a}$ Electrical Engineering Department, The Petroleum Institute, Abu Dhabi, United Arab Emirates; \\ ${ }^{b}$ School of Automation, Southeast University, Key laboratory of Measurement and Control of CSE, \\ Ministry of Education, Nanjing 210096, P.R. China; \\ ${ }^{c}$ Department of Aeronautical and Automotive Engineering, Loughborough University, Leicestershire, \\ LE11 3TU, UK
}

(Received 23 November 2011)

\begin{abstract}
In this paper, a robust nonlinear generalized predictive control (GPC) method is proposed by combining an integral sliding mode approach. The composite controller can guarantee zero steady-state error for a class of uncertain nonlinear systems in the presence of both matched and unmatched disturbances. Indeed, it is well known that the traditional GPC based on Taylor series expansions cannot completely reject unknown disturbance and achieve off-set free tracking performance. To deal with this problem, the existing approaches are enhanced by avoiding the use of the disturbance observer and modifying the gain function of the nonlinear integral sliding surface. This modified strategy appears to be more capable of achieving both the disturbance rejection and the nominal prescribed specifications for matched disturbance. Simulation results on a permanent magnet synchronous motor system demonstrate the effectiveness of the proposed approach.
\end{abstract}

Keywords: disturbance rejection; integral sliding mode control (ISMC); Nonlinear generalized predictive control (GPC); unmatched disturbance; robustness

\section{Introduction}

Currently, there is increasing interest in applying sliding mode control in conjunction with nonlinear control approaches to enhance the disturbance rejection capability (Ren, Zhong and Chen 2015). On the other hand, model predictive control (MPC) is considered as one of the most promising approaches in control engineering and a great deal of research is devoted to it. In predictive control theory, an optimization problem is solved, at each sample time, over a finite prediction horizon in order to find a control which causes the system output to reach a target or follow a predefined trajectory. Then, the obtained optimal input is implemented until the next sampling instant, at which point the procedure is repeated with the update of the process measurement (Garcia, Prett and Morari 1989). It is for this reason that this strategy is also called receding horizon control (RHC).

A look at the literature reveals that Discrete Time Linear Model (DTLM) is widely used in the synthesis of the controller because it gives a fast analytical solution of the optimization problem. However, it is well known that most engineering control systems are inherently nonlinear and continuous time in nature and the use of the DTLM possibly leads to undesirable performance. Despite this, in the case of nonlinear systems with slow dynamics, such as chemical processes, predictive control technique based on Discrete Time Nonlinear Model (DTNLM) can be adopted with satisfactory results even if an on-line optimization is required (Courtial and Touré 1998).

*Corresponding author. Email: rerrouis@pi.ac.ae 
Note that MPC have also been successfully applied to nonlinear distributed parameter systems with slow dynamics (Dufour, Couenne and Touré 2003), where numerical dynamic optimization algorithm has been developed for on-line constrained optimization problem resolution. In spite of its practical success with slow processes, the implementation of MPC is still very complicated for systems having fast dynamics, in particular for nonlinear systems, such as adjustable speed drives because of heavy on-line computational burden (Chen et al. 2000). Over the last few years, substantial progress has been made in applying predictive control to nonlinear systems with fast dynamics, and several nonlinear predictive control laws have been proposed to reduce the computational effort. The majority of these works are based on Continuous Time Nonlinear Model (CTNLM) (Soroush and Kravaris 1996, Lu 1994, 1995, 1998, Chen et al. 1999, Feng, O'Reilly and Ballance 2002, Chen et al. 2003a, Chen 2003, Dabo, Chafouk and Langlois 2009).

A methodology for constructing closed-form nonlinear MPC (NMPC) is proposed in Soroush and Kravaris (1996) and $\mathrm{Lu}$ (1994, 1995), where Taylor series expansion is used to build one-step ahead prediction of the future system output. Multi-step ahead prediction approach is introduced in $\mathrm{Lu}$ (1998), with a view to approximate the nonlinear receding horizon control problem. Despite its complexity, it is shown that this approach may lead to good performance in terms of stability where the one-step ahead prediction is not applicable. A shortcoming of these methods is that the closed-loop system is unstable for plants whose input relative degree is higher than four (Chen et al. 2003a). To overcome this drawback, NMPC scheme for a MIMO system, with the same relative degrees, has been obtained by approximating it with Taylor series expansion to any specified order, which is chosen larger than the input relative degree (Chen et al. 2003a). It is shown that the stability property depends on the so-called control order which should be chosen to be larger than zero when the input relative degree is higher than four. This strategy is extended to nonlinear systems with different relative degrees in Chen (2003). In Dabo, Chafouk and Langlois (2009), the cost function is modified with a specific linear term such that the closed-loop stability is guaranteed even if the input relative degree is higher than four. It should be noted that, in the above-mentioned works, the proposed closed-form NMPC is limited to nonlinear affine systems. However, large engineering control systems can be represented by different classes of nonlinear systems. Driven by this observation, Chen (2004) has developed analytic NMPC for general nonlinear system, which differs from the other NMPC by the fact that the controller scheme provides only the optimal input derivative. Thus, the NMPC should be followed by an integral action to compute the optimal control input. Recently, there has been a steadily increasing interest in application of approximate NMPC to nonlinear systems with fast dynamics such as drives and electrical machines (Errouissi et al. 2012a,b).

The main part of MPC is the model used for system behavior prediction. For nonlinear systems with fast dynamics, the prediction model is obtained by using Taylor series expansion (Gawthrop, Demircioglu and Siller-Alcala 1998). However, it was pointed out in $\mathrm{Lu}$ (1995) that such technique cannot completely remove the steady-state error caused by parameter variations and external disturbances. To tackle this problem, the existing GPC can be combined with disturbance observer to improve the closed-loop performance. This strategy is originally proposed in Chen et al. (1999), and has been adopted for many applications (Errouissi and Ouhrouche 2010). Although this strategy is more capable of handling disturbance attenuation for specific applications, it is still not easy to design a disturbance observer for general MIMO nonlinear systems because it is nontrivial to investigate the global stability (Chen et al. 2003a). Furthermore, in order to achieve a good disturbance rejection performance, a disturbance model is required in designing a nonlinear disturbance observer (Yang et al. 2013).

In this paper, the nonlinear GPC, originally developed in Gawthrop, Demircioglu and SillerAlcala (1998) and refined in Chen et al. (1999), is combined with the so-called integral sliding mode control (ISMC), and applied to a class of uncertain nonlinear systems under both matched and unmatched disturbances. As a matter of fact, the composite controller consists of two additive parts: the continuous part, called nominal control and generated by GPC, and the discontinuous part for rejecting the perturbation. The rejection of matched disturbances via switching 
control law for nonlinear systems is quite mature in SMC (Wang, Zong, Dong and Tian 2015, Ullah, Han and Khattak 2015, Zhu and Khayati 2015, Ghafarirad, Rezaei, Sarhan and Mardi 2015, Park and Kim 2013). However, the use of ISMC guarantees good disturbance rejection performance from the initial time instance. As a result, the closed-loop system in the presence of the matched disturbance behaves exactly as the nominal system under nominal control.

The sliding mode design concept was appeared in Utkin (1977) whereas the ISMC was proposed in Utkin and Shi (1996). In the last several years, a number of research works related to this topic have been published e.g., Cao and Xu (2004), Castaños and Fridman (2006), Rubagotti et al. (2011), Yang, Li and Yu (2013), Ginoya, Shendge and Phadke (2014). The majority of the recent works is looking into the design of an appropriate structure of the integral sliding manifold, especially for unmatched disturbance. In Cao and Xu (2004), it was proved that integral sliding surface allows to achieving the nominal prescribed specifications under matched disturbances. The global stability under unmatched disturbance has also been investigated. A suitable sliding manifold is proposed in Castaños and Fridman (2006) and Rubagotti et al. (2011) to guarantee that the equivalent perturbation from unmatched disturbance is not amplified when dealing with the matched disturbance. In Yang, Li and Yu (2013) and Ginoya, Shendge and Phadke (2014), the SMC has been applied in conjunction with a disturbance observer.

The main objective of this work is to construct an integral sliding manifold structure for nonlinear systems with both matched and unmatched disturbances under GPC law. More interesting, the resultant sliding surface for relative-degree-one systems works as a classical proportional integral (PI) controller whereas the simplified sliding surface for relative-degree-two systems has a proportional integral derivative (PID) controller like structure where the time derivative of the output is replaced by the Lie derivative. Such a structure is well known for nonlinear control systems (Seshagiri and Khalil 2001, Khalil 2002). However, the major difference here lies in the method which is used to derive the sliding manifold and the fact that the proposed structure does not require the time derivative of the output. The main contributions of this paper are summarized as follows

(1) Design of robust continuous time predictive control for a class of uncertain nonlinear systems under both matched and unmatched disturbances.

(2) Boundedness of the closed-loop dynamics is guaranteed.

(3) Zero tracking error can be achieved for time-varying matched disturbances.

(4) Zero steady-state error is guaranteed for unmatched disturbance having constant steadystate value.

(5) Nominal performances can be preserved under matched disturbances.

(6) The disturbance model is not required as for the disturbance observer techniques Yang, Li and Chen (2013), Yang et al. (2013).

\section{Nonlinear generalized predictive control (NGPC)}

A multivariable nonlinear system under disturbance considered in this paper is given by

$$
\left\{\begin{array}{l}
\dot{x}=f(x)+g(x) u(t)+\vartheta(x, t) \\
y=h(x) \\
\vartheta(x, t)=\phi(x) b(t)=\psi_{u}(x, t)+\psi_{m}(x, t)
\end{array}\right.
$$

where $x \in \mathbb{R}^{n}$ is the state of the system with initial condition $x(0)=x_{0}, u \in \mathbb{R}^{m}$ is the input, $y \in \mathbb{R}^{m}$ is the output, and $b \in \mathbb{R}^{q}$ is the disturbance with $q \leq n$, while $f \in \mathbb{R}^{n}, g \in \mathbb{R}^{n \times m}$ and $\phi \in \mathbb{R}^{n \times q}$ are known nonlinear functions. $\psi_{m}(x, t)$ and $\psi_{u}(x, t)$ denote the matched and unmatched disturbances, respectively. The functions $f(x)$ and $h(x)$ are assumed to be continuously differentiable a sufficient number of times. The following Assumptions are imposed on the 
system (1)

A1. The zero-dynamics of the nonlinear system are assumed to be stable.

A2. The input relative degree is well defined.

A3. All states are available.

A4. The rank of the vector function $g(x)$ is equal to $m$.

A5. The disturbances $\vartheta(x, t)$ are bounded by known nonlinear functions .

As in Castaños and Fridman (2006) and Rubagotti et al. (2011), the matched disturbances for a nonlinear system (1) under Assumption A4 is given by

$$
\psi_{m}(x, t)=g(x)\left(g^{T}(x) g(x)\right)^{-1} g^{T}(x) \vartheta(x, t)
$$

Similar with Chen et al. (1999) and Gawthrop, Demircioglu and Siller-Alcala (1998), the cost function of GPC is given by

$$
\Im=\frac{1}{2} \int_{0}^{T} e(t+\tau)^{2} d \tau
$$

where $T$ is the predictive time and $e(t)=y_{r}(t)-y(t)$ is the tracking error, with $y_{r}$ represents the reference signal. The optimal control can be obtained from the necessary condition of optimality

$$
\frac{\partial \Im}{\partial u}=0
$$

First, to simplify the controller design, it is assumed that the outputs have the same relative degree $\rho$. Next, to solve the nonlinear optimization problem (3), each predicted term $e(t+\tau)$ is expanded into a $(\rho+r)$ th order Taylor series expansion using the Lie derivative $h(x)$ along a field of vectors $f(x)$. Here, the standard Lie derivative notation is used (Isidori 1995). The control order is denoted by $r$, which is chosen as zero in this work in order to simplify the notation. However all the results presented in this paper can be expended to arbitrary control order (Chen et al. 2003a). The nominal control law is referred to Chen et al. (1999) and Gawthrop, Demircioglu and Siller-Alcala (1998), given by

$$
u_{0}(t)=G^{-1}(x) \sum_{i=0}^{\rho} K_{i}\left(y_{r}^{(i)}(t)-L_{f}^{i} h(x)\right)
$$

where the matrix $G(x)$ is assumed to be invertible due to A2, given by

$$
G(x)=\frac{\partial L_{f}^{\rho-1} h(x)}{\partial x} g(x)=L_{g} L_{f}^{\rho-1} h(x)
$$

with

$$
K_{i}=\frac{(2 \rho+1) \rho ! T^{i-\rho}}{(\rho+i+1) i !} I_{m}, i=0,1, \ldots, \rho
$$

where $I_{m} \in \mathbb{R}^{m \times m}$ is an identity matrix, and $K_{\rho}=I_{m}$. Furthermore, the error dynamics of the closed-loop system is given by

$$
e^{(\rho)}(t)+K_{\rho-1} e^{(\rho-1)}(t)+\cdots+K_{0} e(t)=0
$$


It should be noted that the resultant control is similar to feedback linearization, but the design method/philosophy and the motivation are different. The stability of the closed-loop system can be established as in Chen et al. (2003a). In fact, since the predictive time is positive, the closed-loop system for a nominal nonlinear system (1) satisfying Assumptions A1-A3 under the GPC control is globally exponentially stable if the relative degree $\rho$ is less than or equal to four. Furthermore, for nonlinear systems with high relative degree, the stability of the closed-loop system can be guaranteed by choosing an appropriate higher control order. On the other hand, the predictive time can only be considered as a controller parameter and can be tuned to improve the controller performance because it affects only the rate of convergence. It can also be shown that a small predictive time will result in a large controller gain and in a fast control response.

\section{Robust NGPC via an integral sliding mode approach}

\subsection{Sliding manifold design}

As pointed out in Feng, O'Reilly and Ballance (2002), it is not easy to design a disturbance observer for general MIMO nonlinear systems because it is nontrivial to investigate the global stability. In this work, an alternative approach of integral sliding mode control developed in Rubagotti et al. (2011), is used as a basis for designing a discontinuous control action to reject the disturbance $b(t)$. The integral sliding manifold is defined as

$$
\begin{aligned}
\sigma(x, t)= & p(x)-p\left(x_{0}\right) \\
& -\int_{0}^{t} l(x)\left(f(x)+g(x) u_{0}(x, \tau)\right) d \tau
\end{aligned}
$$

where $p(x) \in \mathbb{R}^{m \times n}$ is a nonlinear function with

$$
l(x)=\frac{\partial p(x)}{\partial x}
$$

representing the Jacobian matrix. The initial condition $x_{0}$ is the state at $t=0$, and $u_{0}$ is the nominal control defined in (5).

Theorem 1: Considering system (1) and noting that $\sigma\left(x_{0}, 0\right)=0$, the sliding surface $\sigma=0$ can be ensured for all $t \geqslant 0$ by choosing the nonlinear function $p(x)$ as follows

$$
p(x)=\sum_{j=0}^{\rho-1} K_{j+1} L_{f}^{j} h(x)
$$

and designing a robust NGPC as

$$
\begin{aligned}
u(t)= & u_{0}(t)-G^{-1}(x) L(x) \\
& \times\left[\frac{\alpha_{1} L_{1}^{T}(x) \sigma}{\left\|L_{1}^{T}(x) \sigma\right\|} \cdots \frac{\alpha_{q} L_{q}^{T}(x) \sigma}{\left\|L_{q}^{T}(x) \sigma\right\|}\right]^{T}
\end{aligned}
$$

where $\alpha_{i}$ is a switching gain selected such that

$$
\alpha_{i}>\left\|b_{i}(t)\right\|_{\infty}, i=1, \ldots, q
$$

$L(x)$ is a matrix with dimensions $m \times q$ composed of a set of column vectors $L_{i}(x)$ with dimensions 
$m \times 1$ as follows

$$
\begin{aligned}
L(x)=l(x) \phi(x) & =\left[L_{1}(x) \cdots L_{n}(x)\right] \\
& =\sum_{j=0}^{\rho-1} K_{j+1} \frac{\partial L_{f}^{j} h(x)}{\partial x} \phi(x)
\end{aligned}
$$

with $u_{0}, G(x), K_{i}$ and $\sigma$ are defined in (5)-(9), respectively, and the norm of $b$ is defined as $\left\|b_{i}(t)\right\|_{\infty}=\sup _{t}\left|b_{i}(t)\right|$.

Proof: The proof is outlined but more details of the mathematical derivations could be found in Appendices. Define a candidate Lyapunov function

$$
V(\sigma)=\frac{1}{2} \sigma^{T} \sigma
$$

Substituting (10) and (11) into the integral sliding surface (9) leads to

$$
\sigma=\bar{\sigma}-\int_{0}^{t}\left[\sum_{j=0}^{\rho-1} K_{j+1} L_{f}^{j+1} h(x)+G(x) u_{0}(x, \tau)\right] d \tau
$$

where

$$
\begin{aligned}
\bar{\sigma} & =p(x)-p\left(x_{0}\right) \\
& =\sum_{j=0}^{\rho-1} K_{j+1} L_{f}^{j} h(x)-\sum_{j=0}^{\rho-1} K_{j+1} L_{f}^{j} h\left(x_{0}\right)
\end{aligned}
$$

Following (12), the time derivative of the sliding surface (16) is given by

$$
\begin{aligned}
\dot{\sigma} & =L(x) b(t)+G(x) u(t)-G(x) u_{0}(t) \\
& =\sum_{i=1}^{q} L_{i}(x) b_{i}(t)-\sum_{i=1}^{q} L_{i}(x) \frac{L_{i}^{T}(x) \sigma}{\left\|L_{i}^{T}(x) \sigma\right\|} \alpha_{i}
\end{aligned}
$$

Invoking (18) and differentiating the Lyapunov function candidate (15) along the trajectory of the closed-loop system gives

$$
\begin{aligned}
\dot{V}(\sigma) & =\sum_{i=1}^{q} \sigma^{T} L_{i}(x) b_{i}(t)-\sum_{i=1}^{q} \frac{\sigma^{T} L_{i}(x) L_{i}^{T}(x) \sigma}{\left\|L_{i}^{T}(x) \sigma\right\|} \alpha_{i} \\
& \leq-\sum_{i=1}^{q}\left\|L_{i}^{T}(x) \sigma\right\|\left(\alpha_{i}-\left\|b_{i}(t)\right\|\right)
\end{aligned}
$$

Equation (19) implies that under the inequality (13), the time derivative of the Lyapunov function is strictly negative for any $\sigma \neq 0$. This means that $\sigma \rightarrow 0$ as $t \rightarrow \infty$. Moreover, since $\sigma\left(x_{0}, 0\right)=0$, the proposed controller (12) under the condition of (14) ensures the sliding surface $\sigma=0$ for all $t \geqslant 0$. 


\subsection{Simplified sliding surface}

The simplified sliding manifold can be obtained by substituting the nominal control $u_{0}(t)$ into the sliding manifold equation given by (15). The result is outlined here but its derivation is provided in Appendices. Substituting (5) into (16) yields

$$
\sigma=-\int_{0}^{t} K_{0} e(\tau) d \tau-\sum_{j=0}^{\rho-1} K_{j+1}\left(y_{r}^{(j)}(t)-L_{f}^{j} h(x)\right)+\sigma_{0}
$$

where

$$
\sigma_{0}=\sum_{j=0}^{\rho-1} K_{j+1}\left(y_{r}^{(j)}(0)-L_{f}^{j} h\left(x_{0}\right)\right)
$$

The resulting sliding surface is based only on the difference between the Lie derivative $h(x)$ along a field of vectors $f(x)$ and the trajectory to be tracked. Nevertheless, it is worth to note that the sliding surface (16) contains only the terms of the nominal control, and then it can be implemented easily without simplification.

The simplified sliding surface for relative-degree-one systems is given by

$$
\sigma=-K_{0} \int_{0}^{t} e(\tau) d \tau-e(t)+e(0)
$$

whereas the simplified sliding surface for relative-degree-two systems behaves as

$$
\sigma=-K_{0} \int_{0}^{t} e(\tau) d \tau-K_{1} e(t)-\dot{y}_{r}(t)+L_{f} h(x)+\sigma_{0}
$$

with

$$
\sigma_{0}=K_{1} e(0)+\dot{y}_{r}(0)-L_{f} h\left(x_{0}\right)
$$

Note that $L_{f} h(x)=\dot{y}$ for relative-degree-two systems with matched disturbances. Therefore, the structure of the simplified sliding surface behaves as a proportional integral derivative (PID) controller.

\subsection{Closed-loop dynamics}

At the sliding surface, the equivalent control $u_{e}(t)$ is obtained by solving the equation

$$
\dot{\sigma}(x, t)=0
$$

Invoking (14), it follows from (18) that

$$
\begin{aligned}
u_{e}(t) & =u_{0}(t)-G^{-1}(x) L(x) b(t) \\
& =u_{0}(t)-G^{-1}(x) l(x) \phi(x) b(t)
\end{aligned}
$$

To determine the system dynamics at the sliding manifold, we use the equivalent control $u_{e}(t)$ into the equation of (1), one obtains

$$
\dot{x}(t)=f(x)+g(x) u_{o}(t)+\bar{u}(x, t)+\vartheta(x, t)
$$


where

$$
\begin{aligned}
\bar{u}(x, t) & =-g(x) G^{-1}(x) l(x) \vartheta(x, t) \\
& =-g(x) G^{-1}(x) l(x)\left(\psi_{u}(x, t)+\psi_{m}(x, t)\right)
\end{aligned}
$$

Noting that $l(x) g(x)=G(x)$ and substituting (2) into (28) gives

$$
\bar{u}(x, t)=-g(x) G^{-1}(x) l(x) \psi_{u}(x, t)-\psi_{m}(x, t)
$$

Using (29), it can be derived from (27) that

$$
\dot{x}(t)=f(x)+g(x) u_{o}(t)+\phi_{e}(x, t)
$$

where

$$
\begin{aligned}
\phi_{e}(x, t) & =-g(x) G^{-1}(x) l(x) \psi_{u}(x, t)+\psi_{u}(x, t) \\
& =\left(I_{n}-g(x) G^{-1}(x) l(x)\right) \psi_{u}(x, t)
\end{aligned}
$$

The term $\phi_{e}(x, t)$ represents the resulting perturbation from the unmatched disturbance whereas $I_{n} \in \mathbb{R}^{n \times n}$ is an identity matrix. To determine the output dynamics at the sliding manifold, we use the equivalent control and the definition of the input relative degree. As explained in the Appendix $D$, one can obtain

$$
K_{0} e(t)+\sum_{j=0}^{\rho-1} K_{j+1}\left(y_{r}^{(j+1)}(t)-\frac{\partial L_{f}^{j} h(x)}{\partial t}\right)=0
$$

Remark 1: If the disturbance contains only the matched one, i.e., $\psi_{u}(x, t)=0_{n \times 1}$, the closed-loop dynamics becomes

$$
\dot{x}(t)=f(x)+g(x) u_{o}(t)
$$

and the tracking error is governed by

$$
e^{(\rho)}(t)+K_{\rho-1} e^{(\rho-1)}(t)+\cdots+K_{0} e(t)=0
$$

Therefore, at the sliding surface, the closed-loop system for matched disturbance, behaves exactly as the the nominal plant under nominal control. Consequently, the influence of the disturbance is completely removed as long as the nominal plant under nominal control is stable, i.e., the input relative degree is less than or equal to four under Assumption A4. Thus, under matched disturbances, the closed-loop system under the composite controller is asymptotically stable, and the nominal prescribed specifications are preserved.

Remark 2: If the disturbances are composed of both matched and unmatched perturbations, it can be concluded from (30) and (31) that the matched one is completely eliminated. However, the unmatched disturbance is multiplied by a state-dependent matrix, and then it may affect the closed-loop stability. In Rubagotti et al. (2011), a design phase of the Jacobian matrix $l(x)$ is defined for a class of uncertain nonlinear systems such that the 2-norm of the equivalent perturbation is equal to that of the original disturbance, avoiding then the amplification of the unmatched disturbance. As pointed out in Rubagotti et al. (2011), such approach cannot compensate the unmatched disturbance from the output. In general, the closed-loop stability of the perturbed system (30) depends on the nature and the size of the equivalent perturbation $\phi_{e}(x, t)$. Following assumptions $\mathrm{A} 1$ and $\mathrm{A} 4$, it is clear that, in the sliding mode, the resulting 
perturbation $\phi_{e}(x, t)$ is bounded. A general design procedure for investigating the stability of perturbed systems can be found in Khalil (1996). In Cao and Xu (2004), the stability result of the closed-loop system under integral sliding mode control is detailed for unmatched disturbance satisfying assumption A5. In principle, the latest technique can be used for global stability analysis in the presence of the bounded disturbance. Accordingly, as the nominal closed-loop system is globally asymptotically stable under nominal control, the stability of (30) only depends on the nature of the equivalent perturbation $\phi_{e}(x, t)$ and its bounding function under the composite controller (12). Furthermore, it can be concluded from (32) that if the internal driven dynamics is stable, the tracking error $e(t)$ is bounded.

Therefore, for a class of uncertain nonlinear systems, satisfying the assumptions A1-A5, the closed-loop system under the composite controller (12) consisting of nonlinear GPC (5) and integral sliding mode (9) is stable. In other words, boundedness of the closed-loop dynamics is guaranteed despite the presence of bounded unknown disturbance (whether matched or unmatched). Furthermore, if we assume that unmatched disturbances satisfy $\lim _{t \rightarrow \infty} \dot{\psi}_{u}(x, t)=0$, then the nonlinear system (30) has a constant steady-state. In this case, it follows from (32) that the tracking error $e(t)$ converges asymptotically to zero as t goes to infinity.

\section{Application to a permanent magnet synchronous motor (PMSM)}

\subsection{Perturbed model of the PMSM}

The dynamic mathematical model of PMSM can be described in the so-called rotating $(d-q)$ reference as in Ha, Kim and Hyun (2015) and Qi, Bao and Shi (2013). The perturbed model of the PMSM in the rotor reference $(d, q)$ can be expressed in the nonlinear affine form of (1) as in Errouissi et al. (2012a), with

$$
f(x)=\left[\begin{array}{c}
-\frac{R}{L_{d}} i_{d}+\frac{L_{q}}{L_{d}} p \omega_{r} i_{q} \\
-\frac{R}{L_{q}} i_{q}-\frac{L_{d}}{L_{q}} p \omega_{r} i_{d}-\frac{\varphi_{v} p \omega_{r}}{L_{q}} \\
\frac{p}{J}\left(\varphi_{v} i_{q}+\left(L_{d}-L_{q}\right) i_{q} i_{d}\right)-\frac{B}{J} \omega_{r}
\end{array}\right]
$$

and

$$
g(x)=\left[\begin{array}{cc}
\frac{1}{L_{d}} & 0 \\
0 & \frac{1}{L_{q}} \\
0 & 0
\end{array}\right] ; \phi(x)=\left[\begin{array}{ccc}
\frac{1}{L_{d}} & 0 & 0 \\
0 & \frac{1}{L_{q}} & 0 \\
0 & 0 & -\frac{1}{J}
\end{array}\right]
$$

The state vector $x$ is composed of the $d$-axis current $i_{d}, q$-axis current $i_{q}$ and the rotor speed $\omega_{r}$. The input vector $u$ consists of the $d$-axis and $q$-axis components $\left[u_{d} u_{q}\right]^{T}$ of the armature voltage. The output vector $y$ consists of the $d$-axis component of the armature current and the rotor speed, that is, $h(x)=\left[h_{1}(x) h_{2}(x)\right]^{T}=\left[i_{d} \omega_{r}\right]^{T} . R, L_{d}, L_{q}$, are the armature resistance, the $d$-axis and $q$-axis inductances. $\varphi_{v}$ is the permanent magnet flux, $p$ is the number of pole pairs, $J$ is the moment of inertia and $B$ is the coefficient of friction.

The actual three phase currents $i_{a}, i_{b}, i_{c}$, and the electrical position $\theta$ are used to compute the $d q$ frame currents as follows

$$
\left[\begin{array}{l}
i_{d} \\
i_{q}
\end{array}\right]=\left[\begin{array}{cc}
\cos (\theta) & \sin (\theta) \\
-\sin (\theta) & \cos (\theta)
\end{array}\right]\left[\begin{array}{ccc}
\frac{\sqrt{2}}{\sqrt{3}} & \frac{-1}{\sqrt{6}} & \frac{-1}{\sqrt{6}} \\
0 & \frac{1}{\sqrt{2}} & \frac{-1}{\sqrt{2}}
\end{array}\right]\left[\begin{array}{c}
i_{a} \\
i_{b} \\
i_{c}
\end{array}\right]
$$


Here, it is assumed that the three phase currents are sinusoidal. This yield

$$
\left\|i_{d}\right\| \leq \sqrt{\frac{3}{2}} I_{M} ;\left\|i_{q}\right\| \leq \sqrt{\frac{3}{2}} I_{M}
$$

From the fact that $\frac{d \theta}{d t}=p \omega_{r}$, the time derivative of the $d q$-axis currents satisfy

$$
\left\|\frac{d i_{d}}{d t}\right\| \leq \sqrt{6} p \omega_{r \max } I_{M} ;\left\|\frac{d i_{q}}{d t}\right\| \leq \sqrt{6} p \omega_{r \max } I_{M}
$$

where $I_{M}$ is the maximum value of the motor current and $\omega_{\text {rmax }}$ is the maximum value of the rotor speed. The disturbances vector $b(t)=\left[\begin{array}{lll}b_{d} & b_{q} & b_{\omega_{r}}\end{array}\right]^{T}$ represent perturbation resulting from parameter variations $\Delta R, \Delta L_{d}, \Delta L_{q}, \Delta \varphi_{v}, \Delta J$ and $\Delta B$, and unknown external disturbances such as load torque $T_{L}$. Note that $\Delta X=X_{t}-X$ with $X_{t}$ represents the actual value and $X$ is the nominal value. Similar to Errouissi et al. (2012a), the disturbances can be expressed as

$$
\begin{aligned}
b_{d} & =\Delta R i_{d}-\Delta L_{q} p \omega_{r} i_{q}+\Delta L_{d} \frac{d i_{d}}{d t} \\
b_{q} & =\Delta R i_{q}+\Delta L_{d} p \omega_{r} i_{d}+\Delta \varphi_{v} p \omega_{r}+\Delta L_{q} \frac{d i_{q}}{d t} \\
b_{\omega_{r}} & =\Delta B \omega_{r}-p\left(\Delta \varphi_{v}+\Delta\left(L_{d}-L_{q}\right) i_{d}\right) i_{q}+T_{L}+\Delta J \frac{d \omega_{r}}{d t}
\end{aligned}
$$

Combining (38), (39) and (40), and noting that the actual phase current cannot exceed its maximal value $I_{M}$ during both transient and steady-state, it can be concluded that the matched disturbances are bounded by

$$
\begin{aligned}
&\left\|b_{d}\right\| \leq\|\Delta R\| \sqrt{\frac{3}{2}} I_{M}+\left\|\Delta L_{q}\right\| \sqrt{\frac{3}{2}} p \omega_{r \max } I_{M} \\
&+\left\|\Delta L_{d}\right\| \sqrt{6} p \omega_{r \max } I_{M} \\
&\left\|b_{q}\right\| \leq\|\Delta R\| \sqrt{\frac{3}{2}} I_{M}+\left\|\Delta L_{d}\right\| \sqrt{\frac{3}{2}} p \omega_{r \max } I_{M} \\
&+\left\|\Delta L_{q}\right\| \sqrt{6} p \omega_{r \max } I_{M}+\left\|\Delta \varphi_{v}\right\| p \omega_{r \max }
\end{aligned}
$$

Since the slope of the actual speed is limited by the maximal acceleration of the drive $\left\|\frac{d \omega_{r}}{d t}\right\|_{\max }=$ $\frac{T_{e \max }}{J_{t}}$, it follows that the unmatched disturbance is bounded by

$$
\begin{array}{r}
\left\|b_{\omega_{r}}\right\| \leq\left\|\frac{\Delta J}{J_{t}}\right\| T_{e \max }+\|\Delta B\| \omega_{r \max }+\left\|T_{L}\right\| \\
+\left\|\Delta \varphi_{v}\right\| p \sqrt{\frac{3}{2}} I_{M}+\left\|\Delta L_{d}-\Delta L_{q}\right\| p \frac{3}{2} I_{M}^{2}
\end{array}
$$

where $T_{e \max }$ represents the maximum torque that the motor can produce, and it is given by the specifications of the motor. Here, $J_{t}$ represents the actual value of the moment of inertia. 


\subsection{Design of the controller}

A nominal nonlinear predictive controller (5) is designed as

$$
u_{0}(t)=G^{-1}(x)\left[\begin{array}{c}
\sum_{i=0}^{1} K_{i}^{\rho_{1}}\left(i_{d r}^{(i)}(t)-L_{f}^{i} h_{1}(x)\right) \\
\sum_{i=0}^{2} K_{i}^{\rho_{2}}\left(\omega_{r r}^{(i)}(t)-L_{f}^{i} h_{2}(x)\right)
\end{array}\right]
$$

where $\omega_{r r}$ represents the speed reference and $i_{d r}$ represents the desired output of the $d$-axis current. It follows from (6) and ( 7) that

$$
K_{0}^{1}=\frac{3}{2 T} ; K_{1}^{1}=1 ; K_{0}^{2}=\frac{10}{3 T^{2}} ; K_{1}^{2}=\frac{5}{2 T} ; K_{2}^{2}=1
$$

and

$$
G(x)=\left[\begin{array}{c}
\frac{1}{L_{d}} \\
\frac{p}{J L_{d}}\left(L_{d}-L_{q}\right) i_{q} \frac{p}{J L_{q}}\left(\varphi_{v}+\left(L_{d}-L_{q}\right) i_{d}\right)
\end{array}\right]
$$

In practice $i_{d}$ cannot be equal to $\frac{\varphi_{v}}{\left(L_{q}-L_{d}\right)}$, thus the matrix $G(x)$ is invertible for all $x \in \mathbb{R}^{3}$. In addition, as each input relative degree is less than four, the nominal closed-loop system under the predictive controller (44) is globally exponentially stable Chen et al. (2003a). As explained above, in the presence of the bounded disturbances, the nominal predictive controller can be combined with integral sliding mode control to remove the steady-state error. Indeed, the resulting robust controller is defined by (12) with $u_{0}(t)$ is given by (44), and the matrix $L(x)$ for PMSM is written as

$$
L(x)=\left[\begin{array}{ccc}
\frac{1}{L_{d}} & 0 & 0 \\
\frac{p\left(L_{d}-L_{q}\right) i_{q}}{J L_{d}} & \frac{p\left(\varphi_{v}+\left(L_{d}-L_{q}\right) i_{d}\right)}{J L_{q}} & \left(\frac{B}{J^{2}}-\frac{5}{2 T J}\right)
\end{array}\right]
$$

Taking into account the value of the relative degrees, the sliding surface given by (22) is considered for $d$-axis current regulation whereas the sliding surface given by (23)- (24) is adopted for rotor speed regulation.

\subsection{Asymptotic stability analysis}

Corollary: Consider the nonlinear system (1) with the vector functions given by (35) and (36) and suppose that the disturbance $b(t)$ defined by (40) is bounded and satisfying $\lim _{t \rightarrow \infty} \dot{T}_{L}=0$. Then, under the control law (12), the perturbed closed-loop system is globally stable. Additionally, if the reference signal has a constant steady-state value, the system output tracks the desired output with an error which converges to zero as $t$ goes to $\infty$.

proof: Substituting (36) and (47) into (31), under the composite controller, the resulting perturbation from the unmatched disturbance $b_{\omega_{r}}$ becomes as

$$
\phi_{e}(x, t)=\left[0 \frac{\left(\frac{B}{J^{2}}-\frac{5}{2 T J}\right)}{p\left(\varphi_{v}+\left(L_{d}-L_{q}\right) i_{d}\right)}-\frac{1}{J}\right]^{T} b_{\omega_{r}}
$$

According to remark 1, the matched disturbance can be completely rejected under the proposed controller. This implies that the tracking error $e_{d}=i_{d r}-i_{d}$ is asymptotically stable. Following remark 2 , the closed-loop dynamics of the perturbed system only depends on the boundedness of the equivalent perturbation $\phi_{e}(x, t)$ given by (48). 
Table 1. Parameters of the PMSM

\begin{tabular}{l|l}
\hline \hline PMSM & Nominal values \\
\hline Pesistance, $R$ & $1.2 \Omega$ \\
\hline$d$-axis inductance, $L_{d}$ & $0.011 \mathrm{H}$ \\
\hline$q$-axis inductance, $L_{d}$ & $0.011 \mathrm{H}$ \\
\hline Permanent magnet flux, $\varphi_{v}$ & $0.2205 \mathrm{~Wb}$ \\
\hline Number of pole pairs, $p$ & 3 \\
\hline Moment of inertia, $J$ & $0.006 \mathrm{kgm}^{2}$ \\
\hline Coefficient of friction, $B$ & $0.0001 \mathrm{Nm} . \mathrm{s} / \mathrm{rad}$ \\
\hline Nominal load torque, $T_{L}$ & $5 \mathrm{Nm}$ \\
\hline Maximum torque, $T_{e m a x}$ & $10 \mathrm{Nm}$ \\
\hline Nominal phase current, $I_{n}$ & $7 \mathrm{~A}$ \\
\hline Maximum phase current, $I_{M}$ & $14 \mathrm{~A}$ \\
\hline \hline
\end{tabular}

Note that the $d$-axis component can be rewritten as

$$
i_{d}=i_{d r}-e_{d}
$$

By substituting (49) into $\phi_{e}(x, t)$ in $(48)$, it can be shown that $\phi_{e}(x, t)$ only depends on error $e_{d}$, reference $i_{d r}$ and unmatched disturbance $b_{\omega_{r}}$. In practice the $d$-axis current is generally regulated to zero, and the electric current loop is designed with the aim of achieving faster response than the mechanical loop. Therefore, the error $e_{d}$ converges rapidly to zero. Under this condition, the stability of the closed-loop system only depends on the boundedness of the unmatched disturbance $b_{\omega_{r}}$.

As $\lim _{t \rightarrow \infty} \dot{T}_{L}=0$, and the goal is to maintain the system output at a desired steady-state level, rather than tracking fast time-varying references, it can be concluded from (40) that $\lim _{t \rightarrow \infty} \dot{b}_{\omega r}=0$. Thus, according to remark 2 , the tracking error $e_{\omega}=\omega_{r r}-\omega_{r}$ vanishes asymptotically.

\subsection{Simulation results}

Computer simulations have been carried out using the Matlab software package to verify the performances and the effectiveness of the proposed controller regarding robustness. The predictive time is taken as $T=0.005 \mathrm{~s}$. In the controller, the reference $i_{d r}$ must be once differentiable, and the reference $\omega_{r r}$ must be twice differentiable. For PMSM, the $d$-axis current reference is generally set equal to zero. The speed reference trajectory is realized by a second order linear filter with slow dynamics so that the current does not exceed its maximal value during transients. Furthermore, the term $\frac{L_{i}^{T}(x) \sigma}{\left\|L_{i}^{T}(x) \sigma\right\|}$ in the controller (12) is approximated by $\frac{L_{i}^{T}(x) \sigma}{\left|L_{i}^{T}(x) \sigma\right|+1}$, and a linear low-pass filter is applied to the resulting discontinuous control variable for reducing the so-called chattering effect.

The speed reversal at $\pm 100 \mathrm{rad} / \mathrm{s}$ was performed to illustrate the effectiveness of the proposed controller with respect to the speed trajectory tracking. The speed change takes place at $t=0.4 \mathrm{~s}$. To verify the robustness of the proposed control scheme, the actual parameters of the PMSM were modified in the mathematical model at $t=0 s$. Indeed, $R, L_{q}, L_{d}, \varphi_{v}, B$ and $J$ are, respectively, set equal to $50 \%, 60 \%, 70 \%, 120 \%, 50 \%$ and $50 \%$ of their nominal values given in the Table 1 . In addition, the nominal load torque, i.e., $T_{L}=5 \mathrm{Nm}$, is suddenly applied at $t=0.2 \mathrm{~s}$. It is worth to note that the controller is computed using only the nominal values with $T_{L}=0$ and $L_{d}=L_{q}$. However, to guarantee the stability of the closed-loop system, the switching gains $\alpha_{1}, \alpha_{2}$ and $\alpha_{3}$ are obtained using the inequalities (41), (42) and (43). Thus, one can choose $\alpha_{1}=73, \alpha_{2}=81$ and $\alpha_{3}=18$ when $\omega_{\text {rmax }}$ is considered equal to $100 \mathrm{rad} / \mathrm{s}$.

As shown in Fig. 1, the controller has proved to be effective regarding speed trajectory track- 
(a)

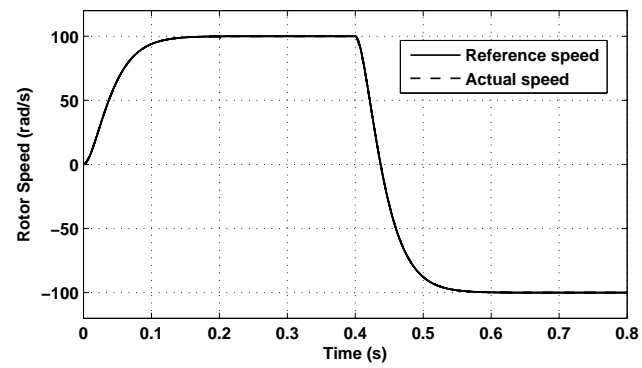

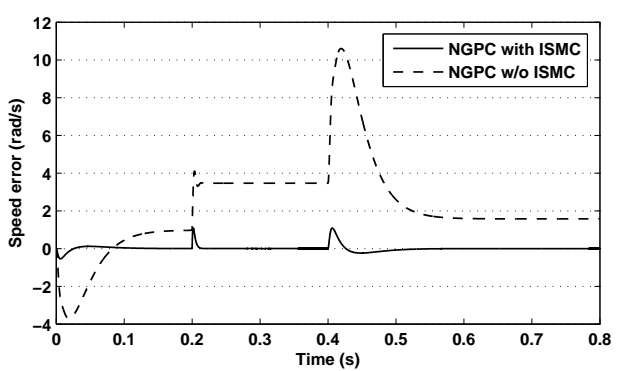

Figure 1. Speed trajectory tracking response and tracking error in the presence of unknown external disturbances and parameter variations under the control laws (5) (solid line) and (12) (dashed line).

(a)

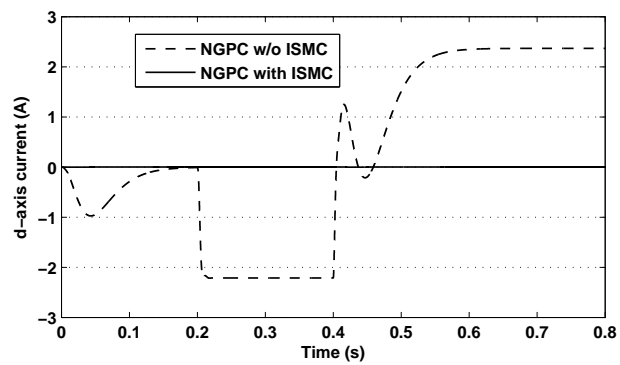

(b)

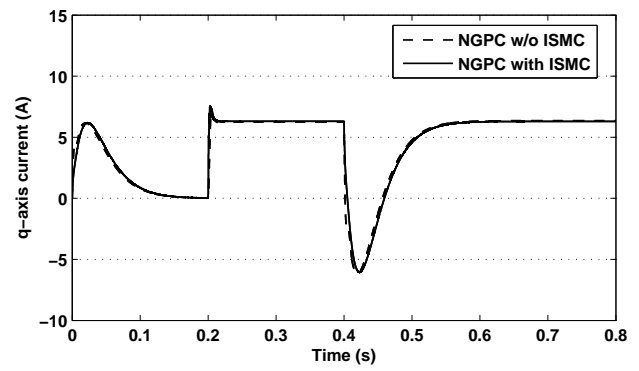

Figure 2. $d q$-axis components of the armature current in the presence of unknown external disturbances and parameter variations under the control laws (5) (solid line) and (12) (dashed line).

(a)

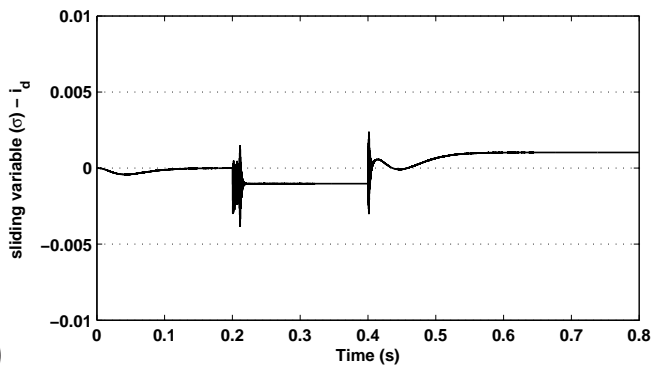

(b)

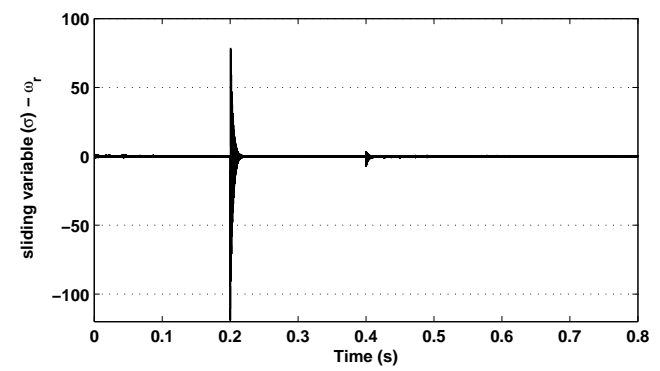

Figure 3. Sliding variables for $d$-axis current and rotor speed

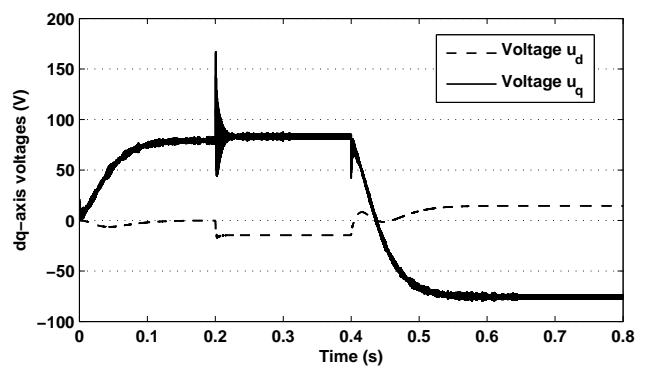

Figure 4. $d q$-axis components of the armature voltages

ing. Figs. 2, 3 and 4 compare the performances of NGPC with and without integral sliding mode control (ISMC). It is clear that the speed error is quickly removed and the $d$-axis component is maintained equal to zero when combining NGPC with ISMC. Thus, both matched and unmatched disturbances are rejected when ISMC is combined with NGPC. From Fig.4, it can be observed that $q$-axis current is satisfactory controlled during transients.

As shown in Fig. 5, the fluctuations of the control variables $u_{d}$ and $u_{q}$ are not significant due to the use of the linear low-pass filter. Figs. 6 and 7 show the two components of the sliding manifold $\sigma$. As expected, after each transient, the sliding manifold is maintained equal to zero. 


\section{Conclusion}

This paper presents robust continuous predictive control for nonlinear systems with fast dynamics. The case where the nonlinear affine system is subjected to either model/plant mismatch or external perturbation is investigated to enhance the robustness of the closed-loop system. To this end, the existing integral sliding mode control is revised and integrated into the controller. For this, a new nonlinear gain function is proposed to design an appropriate structure of the integral sliding manifold for unmatched disturbances. Stability analysis of the composite controller is provided. It is shown that zero tracking error can be achieved in the presence of unknown matched and unmatched disturbances. The proposed approach is applied to PMSM to illustrate its effectiveness regarding speed trajectory tracking, stability, and disturbance rejection.

The main contribution of this paper is that by taking the advantage of the integral sliding manifold, a new composite controller is developed which is able to cope with both matched and mismatched disturbances. Different from the nonlinear disturbance observer technique, there is no knowledge of an explicit model of the disturbance knowledge required. The proposed approach is easy to design and implement in a real-time system. A suitable choice of the gain function in the integral sliding model control is proposed to facilitate the design process. The method proposed in this paper provides practitioners with an alternative method in improving disturbance rejection for nonlinear systems under a wide range of disturbances.

\section{References}

Chen WH, Ballance DJ, Gawthrop PJ, Gribble JJ and O'Reilly J (1999) Nonlinear PID predictive controller. IEE Proceedings-Control Theory and Applications, 146(6):603-611.

Chen WH, Ballance DJ and Gawthrop PJ (2003) Optimal control of nonlinear systems: a predictive control approach. Automatica, 39(4):633-641.

Chen WH, Ballance DJ and O'Reilly J (2000) Model predictive control of nonlinear systems: Computational burden and stability. In IEE Proceedings-Control Theory and Applications, 147: 387-394.

Castaños F and Fridman L (2006) Analysis and design of integral sliding manifolds for systems with unmatched perturbations. IEEE Transactions on Automatic Control, 51(5):853-858.

Chen WH (2003) Closed-form nonlinear mpc for multivariable nonlinear systems with different relative degree. In Proceedings of the 2003 American Control Conference, pp. 4887-4892.

Chen WH (2004) Predictive control of general nonlinear systems using approximation. In IEE Proceedings-Control Theory and Applications, 151: 137-144.

Courtial E and Touré Y (1998) Nonlinear model predictive control: constrained optimization and practical stability. In International Symposium on Nonlinear Model Predictive Control, Ascona, pp. 36.

Cao WJ and Xu JX (2004) Nonlinear integral-type sliding surface for both matched and unmatched uncertain systems. IEEE Transactions on Automatic Control, 49(8):1355-1360.

Dabo M, Chafouk H and Langlois N (2009) Unconstrained ncgpc with a guaranteed closedloop stability: Case of nonlinear siso systems with the relative degree greater than four. In Proceedings of the 48th IEEE Conference on Decision and Control, pp. 1980-1985.

Dufour P, Couenne F and Touré Y (2003) Model predictive control of a catalytic reverse flow reactor. IEEE Transactions on Control Systems Technology, 11(5):705-714.

Errouissi R and Ouhrouche M (2010) Nonlinear predictive controller for a permanent magnet synchronous motor drive. Mathematics and Computers in Simulation, 81(2):394-406.

Errouissi R, Ouhrouche M, Chen WH and Trzynadlowski AM (2012a) Robust cascaded nonlinear predictive control of a permanent magnet synchronous motor with antiwindup compensator. IEEE Transactions on Industrial Electronics, 59(8):3078-3088.

Errouissi R, Ouhrouche M, Chen WH and Trzynadlowski AM (2012b) Robust nonlinear predic- 
tive controller for permanent-magnet synchronous motors with an optimized cost function. IEEE Transactions on Industrial Electronics, 59(7): 2849-2858.

Feng W, O'Reilly J and Ballance DJ (2002) Mimo nonlinear pid predictive controller. IEE Proceedings-Control Theory and Applications, 149(3):203-208.

Gawthrop PJ, Demircioglu H and Siller-Alcala II (1998) Multivariable continuous-time generalised predictive control: A state-space approach to linear and nonlinear systems. In IEE Proceedings-Control Theory and Applications, 145: 241-250.

Garcia CE, Prett DM and Morari M (1989) Model predictive control: theory and practice-A survey. Automatica, 25(3):335-348.

Ginoya D, Shendge PD and Phadke SB (2014) Sliding mode control for mismatched uncertain systems using an extended disturbance observer. IEEE Transactions on Industrial Electronics, 61(4):1983-1992.

Ghafarirad, H, Rezaei, S. M, Sarhan, A. A. D. M, and Mardi, N. A (2015) Modified robust external force control with disturbance rejection with application to piezoelectric actuators. Transactions of the Institute of Measurement and Control, 37(1): 131-143.

Ha, D, Kim, R. Y, and Hyun, D (2015) Internal-model-principle-based robust optimal nonlinear control for position tracking of permanent-magnet synchronous motor servo system. Transactions of the Institute of Measurement and Control, 37(3): 372-381.

Isidori A (1995) Nonlinear Control Systems: An Introduction (3rd edn). Springer-Verlag.

Khalil HK (1996) Nonlinear Systems (2nd edn). Prentice Hall.

Khalil HK (2002) Nonlinear systems (3nd edn). Prentice Hall.

$\mathrm{Lu}$ P (1994) Nonlinear predictive controllers for continuous systems. Journal of Guidance, Control and Dynamics, 17(3): 553-560.

$\mathrm{Lu} \mathrm{P}$ (1995) Optimal predictive control of continuous nonlinear systems. International Journal of Control, 62(3): 633-649.

Lu P (1998) Approximate nonlinear receding-horizon control laws in closed-form. International Journal of Control, 71(1): 19-34.

Li, S, Sun, H, Yang, J, and Yu, X (2015) Continuous finite-time output regulation for disturbed systems under mismatching condition. IEEE Transactions on Automatic Control, 60(1): $277-282$.

Park, T. G, and Kim, D (2014) Design of unknown input observers for linear systems with unmatched unknown inputs. Transactions of the Institute of Measurement and Control, 36(3): 399-410.

Qi, L, Bao, S, and Shi, H (2013) Permanent-magnet synchronous motor velocity control based on second-order integral sliding mode control algorithm. Transactions of the Institute of Measurement and Control, DOI: 0142331213495886

Ren, B, Zhong, Q. C, and Chen, J (2015) Robust Control for a Class of Non-affine Nonlinear Systems Based on the Uncertainty and Disturbance Estimator. IEEE Transactions on Industrial Electronics, DOI: 10.1109/TIE.2015.2421884

Rubagotti M, Estrada A, Castaños F, Ferrara A and Fridman L (2011) Integral sliding mode control for nonlinear systems with matched and unmatched perturbations. IEEE Transactions on Automatic Control, 56(11):2699-2704.

Soroush M and Kravaris C (1996) A continuous-time formulation of nonlinear model predictive control. International Journal of Control, 63(1):121-146.

Seshagiri S and Khalil HK (2001) On introducing integral action in sliding mode control. In Decision and ControlProceedings of the 41 st IEEE Conference on Decision and Control, pp. 1473-1478.

Utkin VI, Guldner J and Shi J (1999). Sliding Mode Control in Electromechanical Systems, CRC press.

Utkin VI and Shi J (1996) Integral sliding mode in systems operating under uncertainty conditions. In Proceedings of the 35th IEEE Decision and Control, pp. 4591-4596.

Utkin VI (1977) Variable structure systems with sliding modes. IEEE Transactions on Automatic 
Control, 22(2):212-222.

Ullah, N, Han, S, and Khattak, M. I (2015) Adaptive fuzzy fractional-order sliding mode controller for a class of dynamical systems with uncertainty. Transactions of the Institute of Measurement and Control, DOI: 0142331215587042.

Wang, F, Zong, Q, Dong, Q, and Tian, B (2015) Disturbance observer-based sliding mode backstepping control for a re-entry vehicle with input constraint and external disturbance. Transactions of the Institute of Measurement and Control, DOI: 0142331215572417.

Yang J, Chen WH, Li S and Chen X (2013) Static disturbance-to-output decoupling for nonlinear systems with arbitrary disturbance relative degree. International Journal of Robust and Nonlinear Control, 23(5): 562-577.

Yang J, Li S, and Chen WH (2013) Nonlinear disturbance observer-based control for multi-input multi-output nonlinear systems subject to mismatching condition. International Journal of Control, 85(8): 1071-1082.

Yang J, Li S and Yu X (2013) Sliding-mode control for systems with mismatched uncertainties via a disturbance observer. IEEE Transactions on Industrial Electronics, 60(1): 160-169.

Zhu, J, and Khayati, K (2015) A new approach for adaptive sliding mode control: Integral/exponential gain law. Transactions of the Institute of Measurement and Control, DOI: 0142331215583328.

Isidori, A. (1995), Nonlinear Control Systems(3rd ed.), New York: Springer-Verlag.

Yoo, D., Yau, S.S.-T., and Gao, Z. (2007), 'Optimal fast tracking observer bandwidth of the linear extended state observer', International Journal of Control, 80, 2007, 102-111. 